\title{
The Ecdysone and Notch Pathways Synergistically Regulate Cut at the Dorsal-Ventral Boundary in Drosophila Wing Discs
}

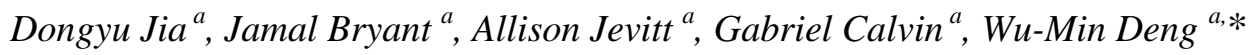

${ }^{a}$ Department of Biological Science, Florida State University, Tallahassee, FL 32306-4370, USA

*Corresponding author.

Tel: +1 850645 1501; fax: +1 850644 0481;

E-mail: wumin@bio.fsu.edu （W.-M. Deng)

(C) 2016. This manuscript version is made available under the Elsevier user license http://www.elsevier.com/open-access/userlicense/1.0/ 


\begin{abstract}
Metazoan development requires coordination of signaling pathways to regulate patterns of gene expression. In Drosophila, the wing imaginal disc provides an excellent model for the study of how signaling pathways interact to regulate pattern formation. The determination of the dorsal/ventral (DV) boundary of the wing disc depends on the Notch pathway, which is activated along the DV boundary and induces the expression of the homeobox transcription factor, Cut. Here, we show that Broad (Br), a zinc-finger transcription factor, is also involved in regulating Cut expression in the DV boundary region. However, Br expression is not regulated by Notch signaling in wing discs, rather, ecdysone signaling is the upstream signal that induces $\mathrm{Br}$ for Cut upregulation. Also, we find that the ecdysone-Br cascade upregulates cut-lacZ expression, a reporter containing a $2.7 \mathrm{~kb}$ cut enhancer region, implying that ecdysone signaling, similar to Notch, regulates $c u t$ at the transcriptional level. Collectively, our findings reveal that the Notch and ecdysone signaling pathways synergistically regulate Cut expression for proper DV boundary formation in the wing disc. Additionally, we show $b r$ promotes Delta, a Notch ligand, near the DV boundary to suppress aberrant high Notch activity, indicating further interaction between the two pathways for DV patterning of the wing disc.

Keywords: ecdysone; Notch; Broad; Cut; Drosophila; wing disc; dorsal-ventral boundary
\end{abstract}




\section{INTRODUCTION}

Metazoan development is strictly and spatiotemporally controlled, by the coordination of multiple signaling pathways for proper gene expression patterns. The Drosophila wing disc provides an excellent model for studying how different signaling pathways interact to induce downstream gene expression that shapes the anterior-posterior (AP) and dorsal-ventral (DV) boundaries (Gonzalez et al., 2006; Restrepo et al., 2014). Notch signaling is active in a narrow strip of cells at the DV boundary of the Drosophila wing disc (de Celis and Garcia-Bellido, 1994; de Celis et al., 1996; Zacharioudaki and Bray, 2014), and regulates the expression of Cut, which is under the regulation of a $2.7 \mathrm{~kb}$ enhancer, for proper wing margin formation (Jack et al., 1991; Neumann and Cohen, 1996). Both Cut and its enhancer cut-lacZ have been used as Notch activity reporters in Drosophila wing discs (Zhang et al., 2014; Zacharioudaki and Bray, 2014).

The Notch pathway is a highly conserved signaling pathway that controls a wide range of developmental processes in metazoans, including cell proliferation and cell fate determination (Klusza and Deng, 2011; Guruharsha et al., 2012). Dysregulation of Notch signaling disrupts normal development, and can cause genetic disorders and cancers in humans (Guruharsha et al., 2012). Therefore, elucidating the cellular and molecular mechanisms of Notch signaling and its associated genetic interactions will advance our understanding of Notch signaling network and shed new light on potential disease treatments.

In the canonical Notch pathway, the Notch receptor, which is located at the cell membrane, is trans-activated by its ligands, Delta and/or Serrate, from neighboring cells. This activation results in proteolytic cleavage of Notch by the gamma-secretase complex to release the intracellular domain of Notch (NICD), which then translocates into the nucleus. Inside the nucleus, NICD forms a trimeric complex with coactivator Mastermind (Mam) and the 
CBF1/Suppressor of Hairless/LAG-1 (CSL) transcription repressor (Suppressor of Hairless $[\mathrm{Su}(\mathrm{H})]$ in Drosophila). The newly formed NICD/Mam/Su(H) complex switches $\mathrm{Su}(\mathrm{H})$ from a repressor to an activator, thus activating the expression of downstream genes (Fortini, 2009; Andersson et al., 2011). Interestingly, ligands expressed from opposing cells (trans-ligands) activate Notch signaling, while ligands from the same cell (cis-ligands) inhibit Notch, which buffer cells against accidental Notch activity (Sprinzak et al., 2010; Palmer et al., 2014).

Broad (Br), a zinc-finger transcription factor, has four different isoforms $\mathrm{Br}-\mathrm{Z1}-\mathrm{Z} 4$ as a result of alternative splicing. Br is an early response gene of the steroid hormone ecdysone, which is essential for the initiation of metamorphosis (Karim et al., 1993; Fletcher and Thummel, 1995), and was recently found as a direct downstream target of Notch signaling during Drosophila oogenesis (Jia et al., 2014). Here, we report that through gain- and loss-of-function studies, $\mathrm{Br}$ is involved in regulating Cut expression in the DV boundary region. Our findings suggest: 1) ecdysone signaling, instead of Notch, is the upstream signal that induces $\mathrm{Br}$ for the upregulation of Cut expression at the DV boundary of the wing disc; 2) both ecdysone (via Br) and Notch signaling work together to transcriptionally regulate Cut expression at the DV boundary in Drosophila wing discs; and 3) Br can promote Delta to suppress aberrant high Notch activity.

\section{Results}

\section{Br regulates Cut at the DV boundary of wing discs.}

Previously, we applied a mouse monoclonal anti-Br-core antibody 25E9 (Emery et al., 1994), and confirmed the ubiquitous expression of $\mathrm{Br}$ in Drosophila wing discs during the late third instar larval stage (Jia et al., 2014). To study the loss-of-function of Br in wing discs, we 
generated $b r-R N A i$ knockdown cells in the posterior compartment of wing discs by the tissuespecific driver en-gal4. The $b r-R N A i$ successfully abolished the expression of $\mathrm{Br}$ in the knockdown cells (Fig. 1A), and the absence of Br led to loss of Cut expression at the DV boundary of wing discs (Fig. 1B). The homozygous clones of a $b r$ amorphic allele, $b r^{n p r-3}$ (Jia et al., 2014) recapitulated the phenotype of Cut absence (Fig. 1C), indicating Br is essential for proper Cut expression. In addition, using the flip-out Gal4/UAS technique (Pignoni and Zipursky, 1997), we found that overexpression of the Br-Z1 isoform in wing discs induced ectopic Cut expression near the DV boundary (Fig. 1D). Through both gain- and loss-of-function studies, we identified that $\mathrm{Br}$ is involved in upregulating Cut expression in the DV boundary region.

\section{Ecdysone signaling regulates $\mathrm{Br}$ to maintain $\mathrm{Cut}$ at the $\mathrm{DV}$ boundary of wing discs.}

Cut is a well-known downstream target of Notch signaling in wing discs, and since $\mathrm{Br}$ is directly upregulated by Notch in follicle cells (Jia et al., 2014), the possibility exists that Notch might regulate Cut via $\mathrm{Br}$. However, we found that induction of $\mathrm{Br}$ expression in wing discs is not regulated by Notch signaling. For instance, in Notch- or mam-RNAi knockdown cells, Cut was downregulated, while Br was properly expressed (Fig. 2A and B) (Jia et al., 2014), indicating that another upstream signal is required for the $\mathrm{Br}$-dependent regulation of Cut expression. $\mathrm{Br}$ has also been cited as an early response gene of the steroid hormone ecdysone (Karim et al., 1993; Fletcher and Thummel, 1995). In this study, we found that ecdysone signaling is indeed the upstream signal to induce $\mathrm{Br}$ for the upregulation of Cut expression at the DV boundary of the wing disc. We applied the EcR B1 dominant negative construct UAS-EcR B1 W650A (Cherbas et al., 2003; Hu et al., 2003; Schubiger et al., 2005; Brown et al., 2006), which encodes an altered EcR B1 protein. This altered EcR B1 loses the ability to bind ligand properly, thus its 
overexpression can act as competitive inhibitors of wild-type EcR B1 and repress ecdysone function. In EcR B1 dominant-negative cells, both Cut and Br were downregulated (Fig. 2C and D), unlike the aforementioned $R N A i$ knockdown of Notch components in cells (Fig. 2A and B). This novel evidence confirms the ecdysone pathway regulates $\mathrm{Br}$ expression, and implies ecdysone acts through Br to maintain Cut for proper DV boundary formation. Additionally, consistent with the previous report that ecdysone is important for the growth and size of wing discs (Mirth et al., 2009; Nijhout and Callier, 2015), the size of the posterior domain with repressed ecdysone function is much smaller (Fig. 2C and D).

\section{Both ecdysone and Notch are required for Cut expression.}

Our results showed that both $\mathrm{Br}$ (mediated by ecdysone) and Notch signaling positively upregulated Cut expression at the DV boundary (Figs. 1B-D and 2A). In Notch signaling defective cells, Cut was downregulated while Br was present (Fig. 2A and B). In the ecdysone defective cells, Cut was downregulated when $\mathrm{Br}$ was absent and Notch activity was upregulated (Figs. 2C, 2D and S1A). These results suggest both ecdysone (via Br) and Notch are needed for proper Cut expression at the DV boundary, and that lacking either eliminates Cut expression.

Previously, Notch signaling was shown to regulate Cut expression via an enhancer region (Jack et al., 1991; Neumann and Cohen, 1996). cut-lacZ expression, a reporter containing the 2.7 $\mathrm{kb}$ cut enhancer region, recapitulated Cut expression at the DV boundary (Jack et al., 1991; Neumann and Cohen, 1996). We thus tested cut-lacZ expression in $b r^{n p r-3}$ mutant clones, and found cut-lacZ was downregulated (Fig. 3A), indicating Br regulates Cut expression at the transcriptional level. Both the trimeric complex NICD/Mam $/ \mathrm{Su}(\mathrm{H})$ and $\mathrm{Br}$, a transcription activation complex and a zinc-finger transcription factor, respectively, can directly bind to DNA 
sequence. One explanation is that both the NICD/Mam/Su(H) transcription activation complex, and $\mathrm{Br}$ or its downstream factors are required to bind to the $2.7 \mathrm{~kb}$ cut enhancer region in order to activate Cut expression.

In addition, overexpression of NICD driven by en-gal4 led to ubiquitous expression of Cut in the posterior compartment (Fig. 3B). Based on our hypothesis that both Notch and ecdysonemediated $\mathrm{Br}$ are required for Cut expression, we reasoned the ubiquitous expression of Cut was partly due to NICD activation, and partly due to the ubiquitous presence of $\mathrm{Br}$. To test the hypothesis, we induced $b r-R N A i$ in NICD-overexpressing posterior compartments, and found Cut was completely absent (Fig. 3C), further confirming that $\mathrm{Br}$ (mediated by ecdysone) and Notch synergize to induce Cut expression in wing discs.

\section{Br promotes Delta levels to suppress aberrant high level of Notch activity.}

Since Notch signaling does not upregulate Cut through $\mathrm{Br}$, we wondered if ecdysone-mediated Br promotes Notch activity to regulate Cut. NRE-GFP (Thompson et al., 2005; Saj et al., 2010) is a Notch-specific reporter, which consists of a Notch responsive element fused with a GFP reporter. Its expression is restricted to the DV boundary. Surprisingly, we found that the NRE-

GFP was highly upregulated in both $b r-R N A i$ knockdown and $b r^{n p r-3}$ mutant clones (Fig. 4A and B), indicating Br expression actually suppresses Notch activity. Subsequent experiments showed Delta levels near the DV boundary decreased in $b r-R N A i$ knockdown cells (Fig. 4C), suggesting $\mathrm{Br}$ is important for proper maintenance of Delta to regulate Notch activity. Consistent with lossof-Br results, NRE-GFP was upregulated in Delta-RNAi knockdown cells at the DV boundary (Fig. 4D), confirming endogenous Delta expression is required to inhibit aberrant high level of Notch activity. Moreover, overexpression of Delta has previously been reported to suppress 
Notch activity at the DV boundary (Fig. 4E) (de Celis and Bray, 1997; Huppert et al., 1997). At the DV boundary, epistatic analysis showed that overexpression of Delta in $b r-R N A i$ knockdown cells caused downregulation of NRE-GFP (Fig. 4F), similar to the reduced NRE-GFP phenotype in Delta-overexpressing cells (Fig. 4E), thus confirming Delta acts downstream of Br to regulate Notch activity. Taken together, we conclude $\mathrm{Br}$ is critical for the proper maintenance of Delta levels that suppress aberrant high Notch activity.

\section{DISCUSSION}

In summary, our results demonstrate that ecdysone (via $\mathrm{Br}$ ) and canonical Notch signaling control Cut expression through the cut enhancer region at the DV boundary. In addition, $\mathrm{Br}$ promotes Delta near the DV boundary to inhibit aberrant Notch activation (Fig. 4G). The steroid hormone, ecdysone, induces significant developmental transitions and exerts pivotal biological functions in Drosophila, including molting, metamorphosis, oogenesis and wing development (Schubiger et al., 1998; Buszczak et al., 1999; Mirth et al., 2009), and even memory and sleep (Ishimoto and Kitamoto, 2011). The development of the Drosophila wing disc is a delicate and well-organized process involving many signaling pathways and factors, such as ecdysone, Notch and Hedgehog signaling, and Cut (Jack et al., 1991; de Celis and Garcia-Bellido, 1994; Mullor et al., 1997). The role of ecdysone has been well studied in late third instar larval and prepupal stages, however, in early- or mid-third instar larval stages, little is known (Thummel, 1996). Br has been suggested as an ecdysone downstream factor that promotes post-critical weight development in third instar lavae (DiBello et al., 1991; Mirth et al., 2009), and is detectable in wing discs at low levels five hours after entering the third instar larval stage, which increases to a high dosage later in the third instar (Mirth et al., 2009). Our results demonstrate $\mathrm{Br}$ is regulated 
by ecdysone signaling, and its induced high dosage later in the third instar potentiates Notch to trigger the expression of Cut. This potentiation by $\mathrm{Br}$ is further supported by the fact that other Notch downstream targets, such as E(spl)bHLH and Wingless, appear in the early third instar larval stage (0-5 h), while Cut responds late, emerging $15 \mathrm{~h}$ after third instar ecdysis (de Celis et al., 1996; Mirth et al., 2009). In addition, overexpression of $\mathrm{Br}$ in wing discs induces ectopic Cut expression near the DV boundary (Fig. 1D), confirming that high dosage of Br indeed can expand the Notch influence zone. A previous study found that the canonical Notch signaling transcription factor, $\mathrm{Su}(\mathrm{H})$, regulates Cut via the cut enhancer (Neumann and Cohen, 1996). Our model (Fig. 4G) proposes both $\mathrm{Br}$ and $\mathrm{NICD} / \mathrm{Mam} / \mathrm{Su}(\mathrm{H})$ are required for the expression of Cut, and that the regulation probably happens at the transcriptional level. At the transcriptional activation level, canonical Notch signaling mediated-NICD recruits coactivator Mam and several cofactors, such as SMRT-related and ecdysone receptor interacting factor (SMRTER), to expel $\mathrm{Su}(\mathrm{H})$-bound co-repressors (Hairless, Groucho, $\mathrm{CtBP}$ ). This process switches $\mathrm{Su}(\mathrm{H})$ from a repressor to an activator of downstream genes (Lipikorn et al., 2010; Heck et al., 2012). Ecdysone-mediated $\mathrm{Br}$ might play a role in the interactions between cofactors to regulate $\mathrm{Cut}$ expression, while another possibility is that $\mathrm{Br}$ and/or its downstream factors attach to the $2.7 \mathrm{~kb}$ cut enhancer region, whereby that anchorage serves as a prerequisite for Notch-mediated Cut expression.

Interestingly, we also found $\mathrm{Br}$ maintains Delta near the DV boundary to inhibit aberrant Notch activity (Fig. 4G). As one of the two ligands that activate Notch signaling, the presence, location, and dosage of Delta play a critical role in development. Two stripes of Delta expressing cells flank the DV boundary, which inhibit aberrant high levels of Notch through cis-inhibition. Meanwhile, high Notch activity induces Cut at the DV boundary that also prevents the 
expression of Delta (de Celis and Bray, 1997). Essentially, high levels of Delta and Notch are mutually exclusive around the DV boundary. However, this mutual exclusivity does not apply in other regions of the wing disc. For instance, overexpression of Delta inhibits Notch activity at the DV boundary, whereas it enhances Notch elsewhere in the wing discs (Fig. 4E) (de Celis and Bray, 1997; Huppert et al., 1997). We speculate that Notch, ecdysone signaling and other DV boundary-located factors, such as Cut, Wingless and Senseless, maintain the complex signaling network around the DV boundary. It is known that cellular and tissue context determines whether Notch can act as an oncogene or a tumor suppressor, which have completely opposite roles in regulating cell proliferation. Further studies in the Drosophila wing disc will shed new light on deciphering the mechanism of this context-dependence.

\section{MATERIALS AND METHODS}

\section{Fly Strains and Genetics}

Strains contain FRT 19A $\mathrm{br}^{n p r-3}$ and $h s F L P, F R T$ 19A RFP (Jia et al., 2014). The following transgenic lines were used in our study: en-Gal4, UAS-mRFP, NRE-GFP (Bloomington Drosophila Stock Center, BDSC\#30729, USA), en-Gal4, UAS-mRFP, NRE-GFP; Gal80ts, UAS-br RNAi (BDSC\#27272), UAS-mam RNAi (BDSC\#28046), UAS-EcR B1 W650A (BDSC\#6872), UAS-br-Z1 (Zhou and Riddiford, 2002), UAS-Delta RNAi (BDSC\#34322), UASDelta (BDSC\#5614), UAS-NICD (Xie et al., 2014), cut-lacZ (Jack et al., 1991) and hsFLP; actin $<C D 2<$ Gal4,UAS-RFP/TM3,Sb.

For the FLP/FRT clone induction (Golic and Lindquist, 1989; Xu and Rubin, 1993), hsFLP, $19 \mathrm{~A} R F P$ was applied, and clones were induced by one session of heat shock for $2 \mathrm{~h}$ at $37^{\circ} \mathrm{C}$ during late second instar larval stage. To generate mosaic wing discs expressing $U A S$ constructs, 
the flip-out Gal4 (Pignoni and Zipursky, 1997) stock hsFLP;actin<CD2<Gal4,UAS-

$R F P / T M 3, S b$ was applied. Flip-out clones were induced by 30 min heat shock at $37^{\circ} \mathrm{C}$ two days before dissection. en-Gal4,UAS-mRFP, NRE-GFP; Gal80ts was used to manipulate gene expression in the posterior compartment of wing discs. In this technique, en-Gal4,UAS-mRFP, NRE-GFP; Gal80ts flies were crossed with $U A S$ lines, and the progeny were raised at $18^{\circ} \mathrm{C}$ until late second instar larval stage, then shifted to $29^{\circ} \mathrm{C}$ for two days before dissection.

\section{Immunohistochemistry}

Immunohistochemistry was carried out as previously described in Drosophila ovaries (Jia et al., ,2014, 2015). The following antibodies were used: mouse anti-Br-Core (25E9) 1:30, antiCut (2B10) 1:15 (Development Studies Hybridoma Bank, USA), rabbit anti- $\beta$-Galactosidase 1:5000 (Sigma, USA). Corresponding Alexa Fluor secondary antibodies (1:400; Invitrogen, USA) were selected according to primary antibodies.

\section{Image analysis}

Images were acquired with a Zeiss LSM 510 confocal microscope and processed in Photoshop and Image J. To quantify the expression levels of both NRE-GFP and Delta in the experiments, images in Fig. 4 were extracted and processed in Image J. In brief, three random areas in the control and manipulated groups at the DV boundary were selected to measure the "mean gray value" by applying the "analyze" menu in Image J. The values of manipulated groups were normalized to those of control groups in order to demonstrate fold changes of expression levels. The statistical analyses were performed using GraphPad Prism 6. Data were expressed as the 
mean \pm SEM. Intergroup differences were assessed by Student's $t$-test. Statistical significance is denoted with asterisks in the figures. $* P<0.05$; ** $P<0.01$; *** $P<0.001$.

\section{ACKNOWLEDGMENTS}

We would like to thank the Molecular Cloning Facility and the Biological Science Imaging Facility at Florida State University for technical help; the DSHB, the TRiP at Harvard Medical School (NIH/NIGMS R01-GM084947) and the Bloomington Stock Center for providing us antibodies and fly stocks; Yi-Chun Huang for critical reading of, and comments on, the manuscript. Special thanks to other members of the Deng lab for technical help and discussions on this project. D.J. is supported by Dissertation Research Grant Award from Florida State University. W.-M. D. is supported by the NIH grant (R01GM072562) and National Science Foundation (IOS-1052333).

\section{SUPPLEMENTARY DATA}

Fig. S1 Ecdysone inhibits Notch activity.

\section{Figure Legends}

Fig. 1. Br regulates the expression of Cut in wing discs.

A-B": Knockdown of $\mathrm{Br}$ in the posterior compartment of the wing disc (en-Gal4 driven UAS-br $R N A i$, marked with RFP, red in $\mathbf{A}$ and $\mathbf{B}$, white in $\mathbf{A}^{\prime}$ and $\mathbf{B}^{\prime}$ ) abolished $\mathrm{Br}$ expression (green in $\mathbf{A}$, white in $\mathbf{A}^{\prime \prime}$ ), and caused the loss of Cut expression (green in $\mathbf{B}$, white in $\left.\mathbf{B}^{\prime \prime}\right)$. C: $b r^{n p r-3}$ 
mutant clones (marked by absence of RFP, indicated by arrowheads) showed no expression of Cut (green in $\mathbf{C}$, white in $\mathbf{C}^{\prime \prime}$ ). D: In Br-Z1 overexpressing cells (marked by RFP, indicated by arrowheads, red in $\mathbf{D}$, white in $\mathbf{D}^{\prime}$ ), Cut (green in $\mathbf{D}$, white in $\mathbf{D}^{\prime \prime}$ ) was induced near the DV boundary. Wing discs are aligned ventral to the top of the image, dorsal to the bottom, posterior to the right. Bars, $20 \mu \mathrm{m}$.

Fig. 2. Br is regulated by ecdysone signaling, but not Notch.

A-B": Knockdown of Mam in the posterior compartment of the wing disc (en-Gal4 driven UAS-mam RNAi, marked with RFP, red in $\mathbf{A}$ and $\mathbf{B}$, white in $\mathbf{A}^{\prime}$ and $\mathbf{B}^{\prime}$ ) abolished Cut expression (green in $\mathbf{A}$, white in $\mathbf{A}^{\prime \prime}$ ), while retaining proper $\mathrm{Br}$ expression (green in $\mathbf{B}$, white in $\left.\mathbf{B}^{\prime \prime}\right)$. C-D": Overexpression of a dominant negative EcR B1 $\left(\mathrm{EcR} B 1^{\mathrm{DN}}\right)$ in the posterior compartment of the wing disc (en-Gal4 driven $U A S-E c R B 1^{D N}$ W650A, marked with RFP, red in $\mathbf{C}$ and $\mathbf{D}$, white in $\mathbf{C}^{\prime}$ and $\mathbf{D}^{\prime}$ ) abolished both Cut (green in $\mathbf{C}$, white in $\mathbf{C}^{\prime \prime}$ ) and Br expression (green in $\mathbf{D}$, white in $\mathbf{D}^{\prime \prime}$ ). Wing discs are aligned ventral to the top of the image, dorsal to the bottom, posterior to the right. Bars, $20 \mu \mathrm{m}$.

Fig. 3. Both ecdysone and Notch signaling are required for Cut expression.

A-A": $b r^{n p r-3}$ mutant clones (marked by absence of RFP, indicated by arrowheads) showed downregulated cut-lacZ reporter (green in A, white in A"). B-B": Overexpression of NICD (enGal4 driven $U A S-N I C D$, marked with RFP, red in $\mathbf{B}$, white in $\mathbf{B}^{\prime}$ ) upregulated Cut in the whole posterior compartment of the wing disc (green in $\mathbf{B}$, white in $\left.\mathbf{B}^{\prime \prime}\right)$. $\mathbf{C}-\mathbf{C}^{\prime \prime}$ : Overexpression of NICD in the Br knockdown cells in the posterior compartment of the wing disc (en-Gal4 driven $U A S-N I C D$ and br RNAi, marked with RFP, red in $\mathbf{C}$, white in $\mathbf{C}^{\prime}$ ) abolished Cut expression 
(green in $\mathbf{C}$, white in $\mathbf{C}^{\prime \prime}$ ). Wing discs are aligned ventral to the top of the image, dorsal to the bottom, posterior to the right. Bars, $20 \mu \mathrm{m}$.

Fig. 4. Br promotes Delta near the DV boundary to suppress aberrant high Notch activity. A-A"': $b r^{n p r-3}$ mutant clones (marked by absence of RFP, indicated by arrowheads) showed elevated levels of NRE-GFP (green in $\mathbf{A}$, white in $\mathbf{A}^{\prime \prime}$ ), and this elevation is statistically significant (A' $\left.\mathbf{A}^{\prime \prime \prime}\right)$. B-C $\mathbf{C}^{\prime \prime \prime}$ : Knockdown of $\mathrm{Br}$ in the posterior compartment of the wing disc (enGal4 driven $U A S$-br RNAi, marked with RFP, red in $\mathbf{B}$ and $\mathbf{C}$, white in $\mathbf{B}^{\prime}$ and $\mathbf{C}^{\prime}$ ) enhanced NRE-GFP expression (green in $\mathbf{B}$, white in $\mathbf{B}^{\prime \prime}$ ), and caused the loss of proper Delta expression (green in $\mathbf{C}$, white in $\mathbf{C}^{\prime \prime}$ ) flanking the DV boundary. The enhancement of NRE-GFP and the downregulation of Delta are statistically significant ( $\mathbf{B}^{\prime \prime \prime}$ and $\left.\mathbf{C}^{\prime \prime \prime}\right)$. D-D"': Knockdown of Delta in the posterior compartment of the wing disc (en-Gal4 driven UAS-Delta RNAi, marked with RFP, red in $\mathbf{D}$, white in $\mathbf{D}^{\prime}$ ) enhanced NRE-GFP expression (green in $\mathbf{D}$, white in $\mathbf{D}^{\prime \prime}$ ), and this enhancement is statistically significant $\left(\mathbf{D}^{\prime \prime \prime}\right)$. E-E': Overexpression of Delta in the posterior compartment of the wing disc (en-Gal4 driven UAS-Delta, marked with RFP, red in $\mathbf{E}$, white in $\mathbf{E}^{\prime}$ ) significantly downregulated NRE-GFP expression at the DV boundary (green in $\mathbf{E}$, white in $\left.\mathbf{E}^{\prime \prime}\right)$. F-F"': Overexpression of Delta in the Br knockdown cells in the posterior compartment of the wing disc (en-Gal4 driven UAS-Delta and $b r R N A i$, marked with RFP, red in $\mathbf{F}$, white in $\mathbf{F}^{\prime}$ ) significantly downregulated NRE-GFP expression at the DV boundary (green in $\mathbf{F}$, white in $\mathbf{F}^{\prime \prime}$ ). Areas circled by dotted lines are DV boundary areas $(\mathbf{B}, \mathbf{D}-\mathbf{F})$, while dotted line in $\mathbf{C}$ separates DV boundary. NRE-GFP expression intensity within DV boundary areas (A, B, D-F) and Delta expression flanking DV boundary are measured. Anterior/posterior compartments are separated by straight lines (B-F). Wing discs are aligned ventral to the top of the image, dorsal to the 
bottom, anterior to the left, posterior to the right. $\mathrm{G}$ : $\mathrm{Br}$ (mediated by ecdysone) and canonical Notch signaling $(v i a \mathrm{Su}(\mathrm{H}))$ control Cut expression through the cut enhancer region at the DV boundary. In addition, Br promotes Delta near the DV boundary to inhibit aberrant Notch activation. ${ }^{*} P<0.05 ; * * P<0.01 ; * * * P<0.001$. Bars, $20 \mu \mathrm{m}$.

Fig. S1. Ecdysone inhibits Notch activity.

A-A": Overexpression of a dominant negative EcR B1 $\left(\mathrm{EcR} \mathrm{B}^{\mathrm{DN}}\right)$ in the posterior compartment of the wing disc (en-Gal4 driven UAS-EcR B1 ${ }^{D N}$ W650A, marked with RFP, red in $\mathbf{A}$, white in $\mathbf{A}^{\prime}$ ) enhanced NRE-GFP expression (green in $\mathbf{A}$, white in $\mathbf{A}^{\prime \prime}$ ). Wing discs are aligned ventral to the top of the image, dorsal to the bottom, posterior to the right. Bars, $20 \mu \mathrm{m}$.

\section{REFERENCES}

Andersson, E.R., Sandberg, R., Lendahl, U., 2011. Notch signaling: simplicity in design, versatility in function. Development 138, 3593-3612.

Brown, H.L., Cherbas, L., Cherbas, P., Truman, J.W., 2006. Use of time-lapse imaging and dominant negative receptors to dissect the steroid receptor control of neuronal remodeling in Drosophila. Development 133, 275-285.

Buszczak, M., Freeman, M.R., Carlson, J.R., Bender, M., Cooley, L., Segraves, W.A., 1999. Ecdysone response genes govern egg chamber development during mid-oogenesis in Drosophila. Development 126, 4581-4589.

Cherbas, L., Hu, X., Zhimulev, I., Belyaeva, E., Cherbas, P., 2003. EcR isoforms in Drosophila: testing tissue-specific requirements by targeted blockade and rescue. Development 130, 271-284.

de Celis, J.F., Bray, S., 1997. Feed-back mechanisms affecting Notch activation at the dorsoventral boundary in the Drosophila wing. Development 124, 3241-3251.

de Celis, J.F., Garcia-Bellido, A., 1994. Roles of the Notch gene in Drosophila wing morphogenesis. Mech. Dev. 46, 109-122.

de Celis, J.F., Garcia-Bellido, A., Bray, S.J., 1996. Activation and function of Notch at the dorsal-ventral boundary of the wing imaginal disc. Development 122, 359-369.

DiBello, P.R., Withers, D.A., Bayer, C.A., Fristrom, J.W., Guild, G.M., 1991. The Drosophila Broad-Complex encodes a family of related proteins containing zinc fingers. Genetics 129, 385-397. 
Emery, I.F., Bedian, V., Guild, G.M., 1994. Differential expression of Broad-Complex transcription factors may forecast tissue-specific developmental fates during Drosophila metamorphosis. Development 120, 3275-3287.

Fletcher, J.C., Thummel, C.S., 1995. The ecdysone-inducible Broad-complex and E74 early genes interact to regulate target gene transcription and Drosophila metamorphosis. Genetics 141, 1025-1035.

Fortini, M.E., 2009. Notch signaling: the core pathway and its posttranslational regulation. Dev. Cell 16, 633-647.

Golic, K.G., Lindquist, S., 1989. The FLP recombinase of yeast catalyzes site-specific recombination in the Drosophila genome. Cell 59, 499-509.

Gonzalez, A., Chaouiya, C., Thieffry, D., 2006. Dynamical analysis of the regulatory network defining the dorsal-ventral boundary of the Drosophila wing imaginal disc. Genetics 174, 1625-1634.

Guruharsha, K.G., Kankel, M.W., Artavanis-Tsakonas, S., 2012. The Notch signalling system: recent insights into the complexity of a conserved pathway. Nat. Rev. Genet. 13, 654-666.

Heck, B.W., Zhang, B., Tong, X., Pan, Z., Deng, W.M., Tsai, C.C., 2012. The transcriptional corepressor SMRTER influences both Notch and ecdysone signaling during Drosophila development. Biol. Open 1, 182-196.

$\mathrm{Hu}, \mathrm{X}$., Cherbas, L., Cherbas, P., 2003. Transcription activation by the ecdysone receptor (EcR/USP): identification of activation functions. Mol. Endocrinol. 17, 716-731.

Huppert, S.S., Jacobsen, T.L., Muskavitch, M.A., 1997. Feedback regulation is central to DeltaNotch signalling required for Drosophila wing vein morphogenesis. Development 124, 3283-3291.

Ishimoto, H., Kitamoto, T., 2011. Beyond molting--roles of the steroid molting hormone ecdysone in regulation of memory and sleep in adult Drosophila. Fly (Austin) 5, 215-220.

Jack, J., Dorsett, D., Delotto, Y., Liu, S., 1991. Expression of the cut locus in the Drosophila wing margin is required for cell type specification and is regulated by a distant enhancer. Development 113, 735-747.

Jia, D., Huang, Y.C., Deng, W.M., 2015. Analysis of Cell Cycle Switches in Drosophila Oogenesis. Methods Mol. Biol. 1328, 207-216.

Jia, D., Tamori, Y., Pyrowolakis, G., Deng, W.M., 2014. Regulation of broad by the Notch pathway affects timing of follicle cell development. Dev. Biol. 392, 52-61.

Karim, F.D., Guild, G.M., Thummel, C.S., 1993. The Drosophila Broad-Complex plays a key role in controlling ecdysone-regulated gene expression at the onset of metamorphosis. Development 118, 977-988.

Klusza, S., Deng, W.M., 2011. At the crossroads of differentiation and proliferation: precise control of cell-cycle changes by multiple signaling pathways in Drosophila follicle cells. Bioessays 33, 124-134.

Lipikorn, R., Chunhapongpipat, K., Sirisup, S., Boonklurb, R., Cooharojananone, N., 2010. Medical Image Segmentation Using Modified Level-Set Model with Multi-Scale Gradient* Vector Flow. Comm. Com. Inf. Sc. 123, 49-57.

Mirth, C.K., Truman, J.W., Riddiford, L.M., 2009. The ecdysone receptor controls the postcritical weight switch to nutrition-independent differentiation in Drosophila wing imaginal discs. Development 136, 2345-2353.

Mullor, J.L., Calleja, M., Capdevila, J., Guerrero, I., 1997. Hedgehog activity, independent of decapentaplegic, participates in wing disc patterning. Development 124, 1227-1237. 
Neumann, C.J., Cohen, S.M., 1996. A hierarchy of cross-regulation involving Notch, wingless, vestigial and cut organizes the dorsal/ventral axis of the Drosophila wing. Development 122, 3477-3485.

Nijhout, H.F., Callier, V., 2015. Developmental mechanisms of body size and wing-body scaling in insects. Annu. Rev. Entomol. 60, 141-156.

Palmer, W.H., Jia, D., Deng, W.M., 2014. Cis-interactions between Notch and its ligands block ligand-independent Notch activity. eLife 3, e04415.

Pignoni, F., Zipursky, S.L., 1997. Induction of Drosophila eye development by decapentaplegic. Development 124, 271-278.

Restrepo, S., Zartman, J.J., Basler, K., 2014. Coordination of patterning and growth by the morphogen DPP. Curr. Biol. 24, R245-255.

Saj, A., Arziman, Z., Stempfle, D., van Belle, W., Sauder, U., Horn, T., Durrenberger, M., Paro, R., Boutros, M., Merdes, G., 2010. A combined ex vivo and in vivo RNAi screen for notch regulators in Drosophila reveals an extensive notch interaction network. Dev. Cell 18, 862876.

Schubiger, M., Carre, C., Antoniewski, C., Truman, J.W., 2005. Ligand-dependent de-repression via $\mathrm{EcR} / \mathrm{USP}$ acts as a gate to coordinate the differentiation of sensory neurons in the Drosophila wing. Development 132, 5239-5248.

Schubiger, M., Wade, A.A., Carney, G.E., Truman, J.W., Bender, M., 1998. Drosophila EcR-B ecdysone receptor isoforms are required for larval molting and for neuron remodeling during metamorphosis. Development 125, 2053-2062.

Sprinzak, D., Lakhanpal, A., Lebon, L., Santat, L.A., Fontes, M.E., Anderson, G.A., GarciaOjalvo, J., Elowitz, M.B., 2010. Cis-interactions between Notch and Delta generate mutually exclusive signalling states. Nature 465, 86-90.

Thompson, B.J., Mathieu, J., Sung, H.H., Loeser, E., Rorth, P., Cohen, S.M., 2005. Tumor suppressor properties of the ESCRT-II complex component Vps25 in Drosophila. Dev. Cell 9, 711-720.

Thummel, C.S., 1996. Flies on steroids--Drosophila metamorphosis and the mechanisms of steroid hormone action. Trends Genet. 12, 306-310.

Xie, G., Yu, Z., Jia, D., Jiao, R., Deng, W.M., 2014. E(y)1/TAF9 mediates the transcriptional output of Notch signaling in Drosophila. J. Cell Sci. 127, 3830-3839.

Xu, T., Rubin, G.M., 1993. Analysis of genetic mosaics in developing and adult Drosophila tissues. Development 117, 1223-1237.

Zacharioudaki, E., Bray, S.J., 2014. Tools and methods for studying Notch signaling in Drosophila melanogaster. Methods 68, 173-182.

Zhang, Q., Zhang, Y., Wu, L., Yang, Y., Li, X., Gao, L., Hou, X., Wu, Y., Hou, G., Li, Z., Lin, X., 2014. dBrms1 acts as a positive regulator of Notch signaling in Drosophila wing. J. Genet. Genomics 41, 317-325.

Zhou, X., Riddiford, L.M., 2002. Broad specifies pupal development and mediates the 'status quo' action of juvenile hormone on the pupal-adult transformation in Drosophila and Manduca. Development 129, 2259-2269. 

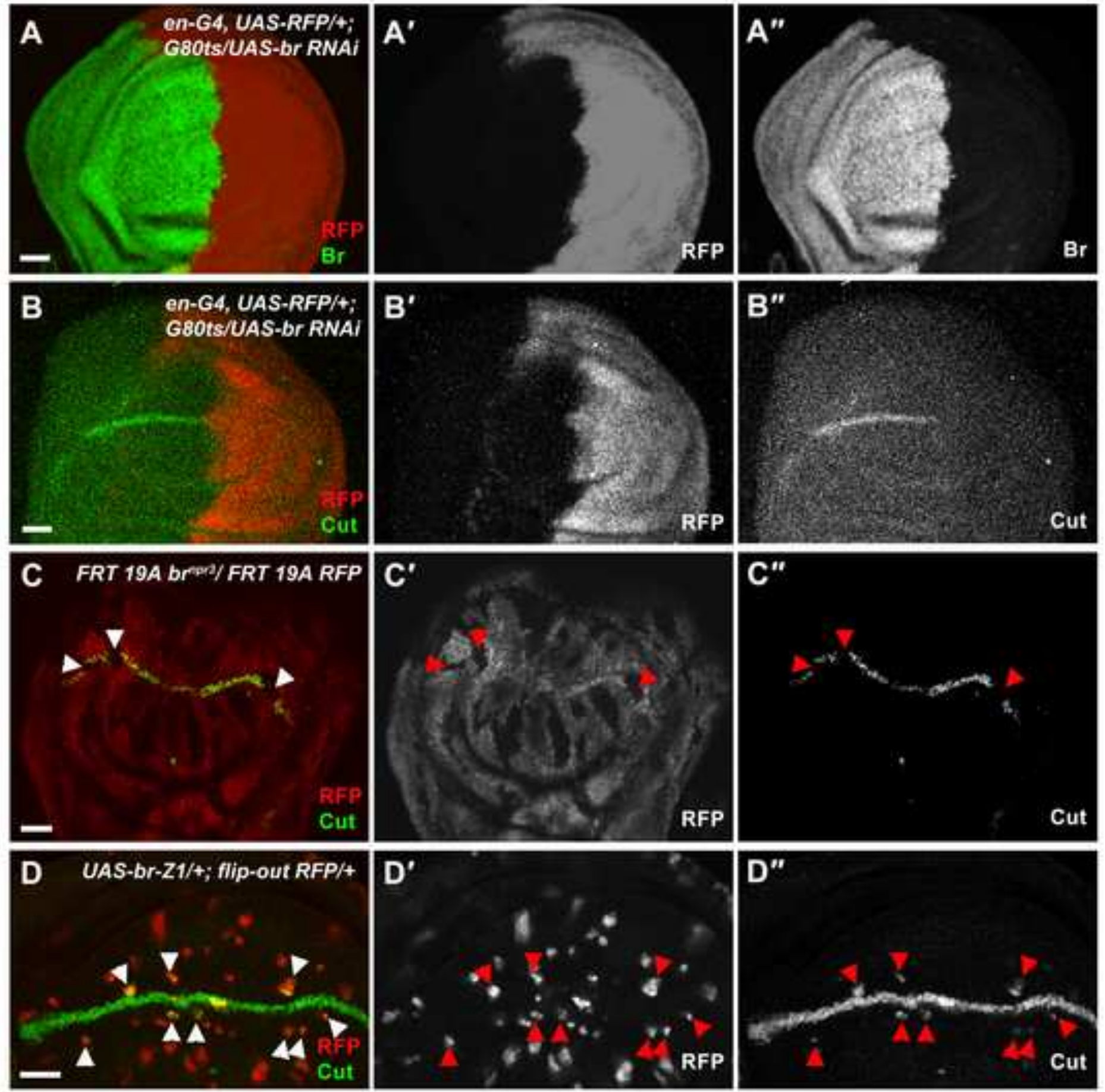

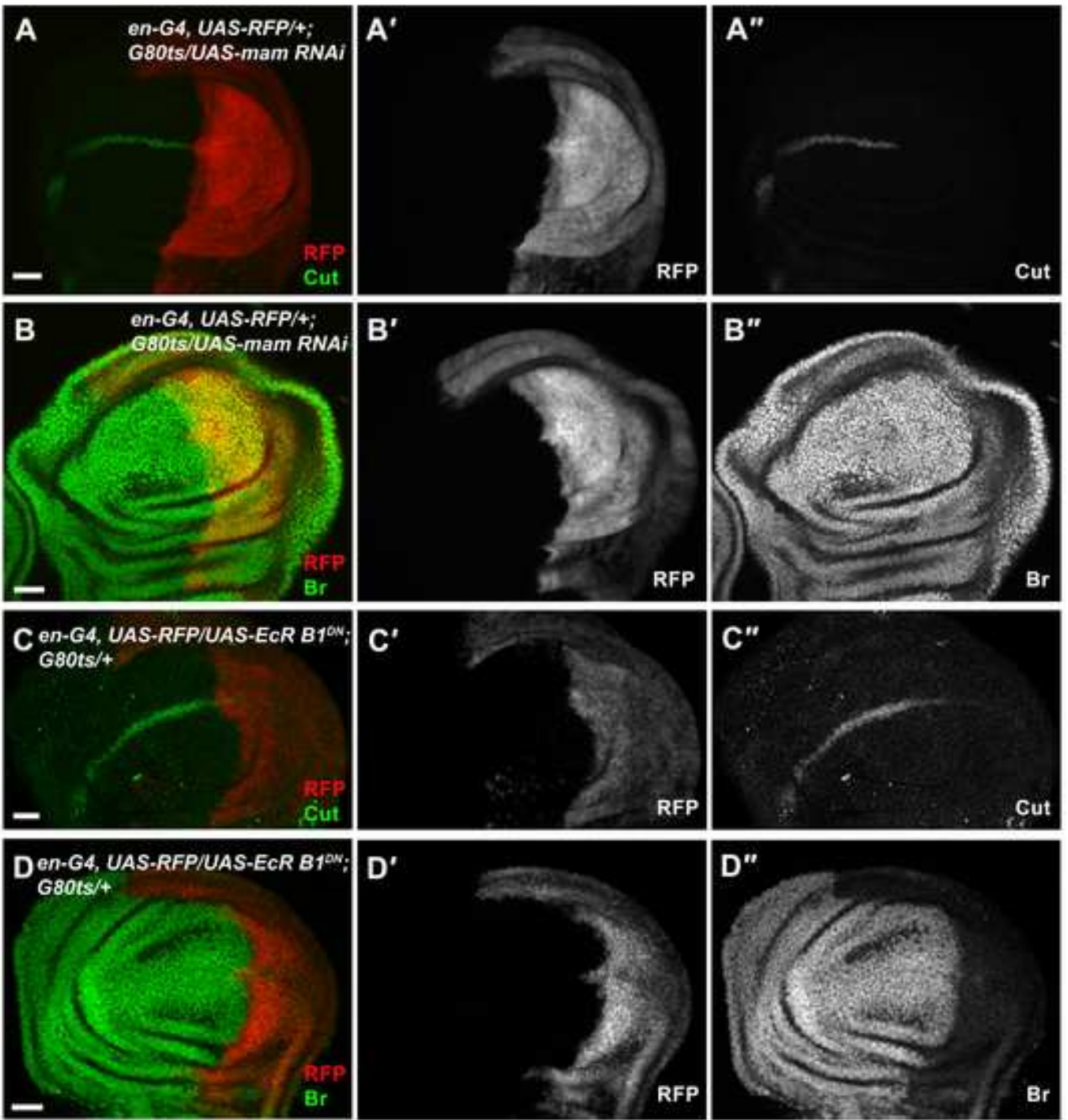


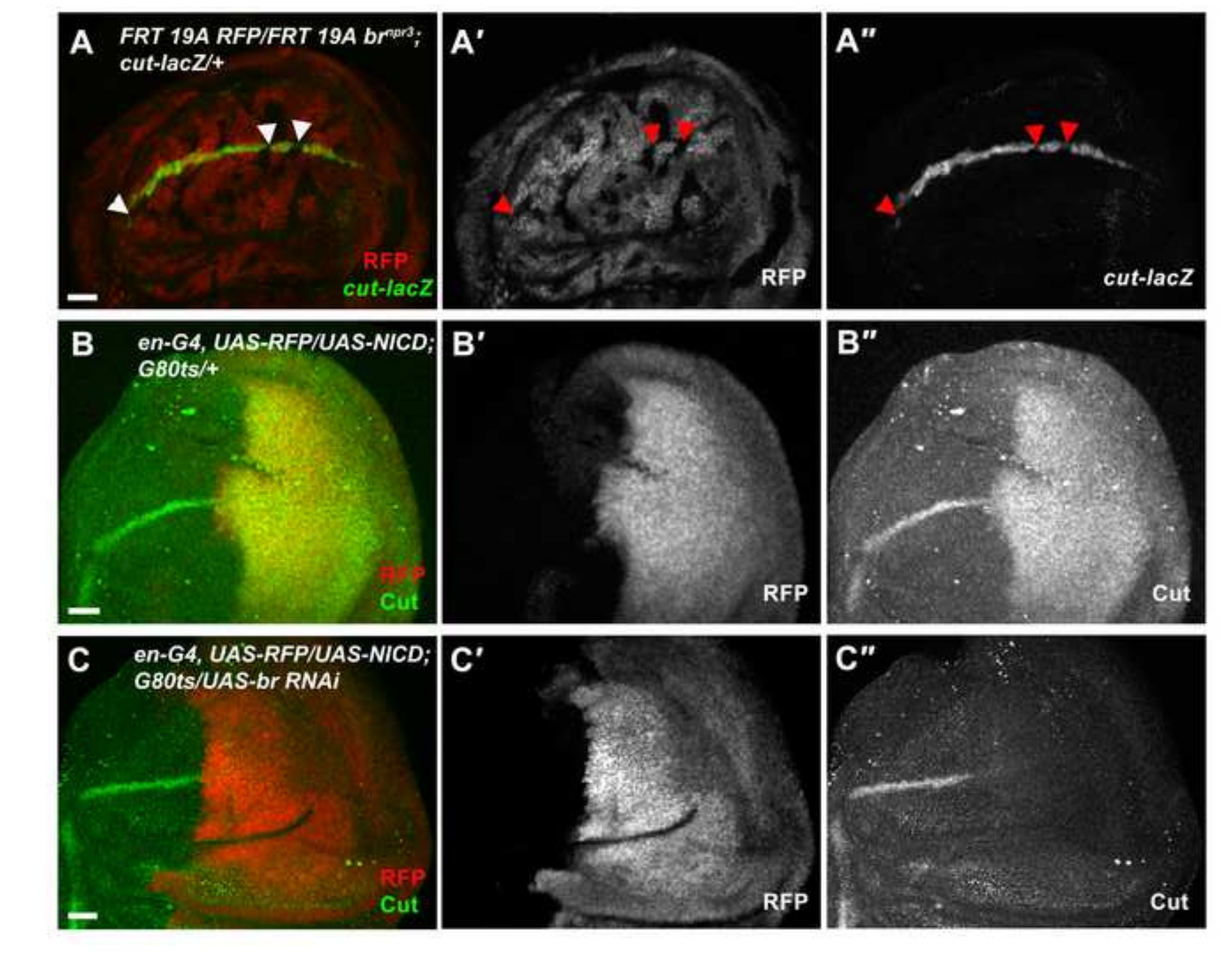



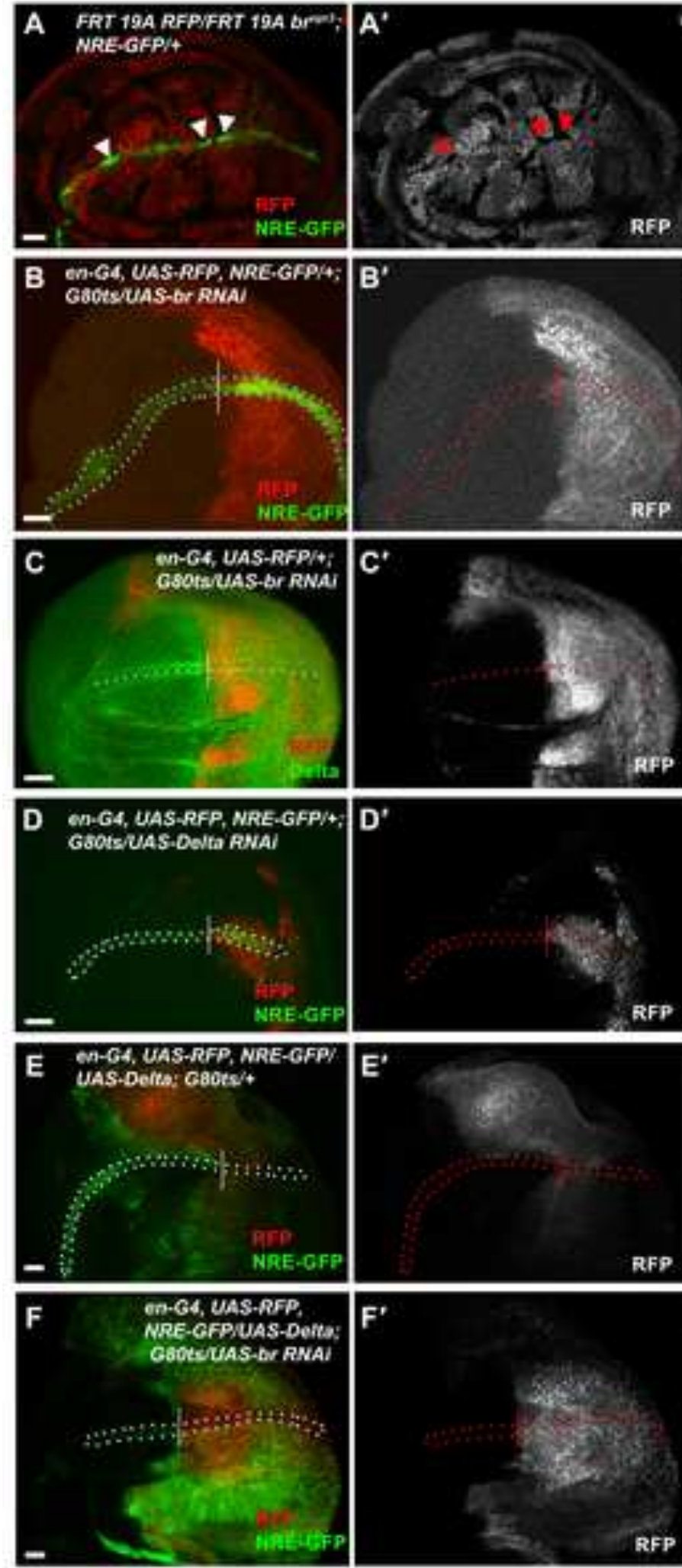

G
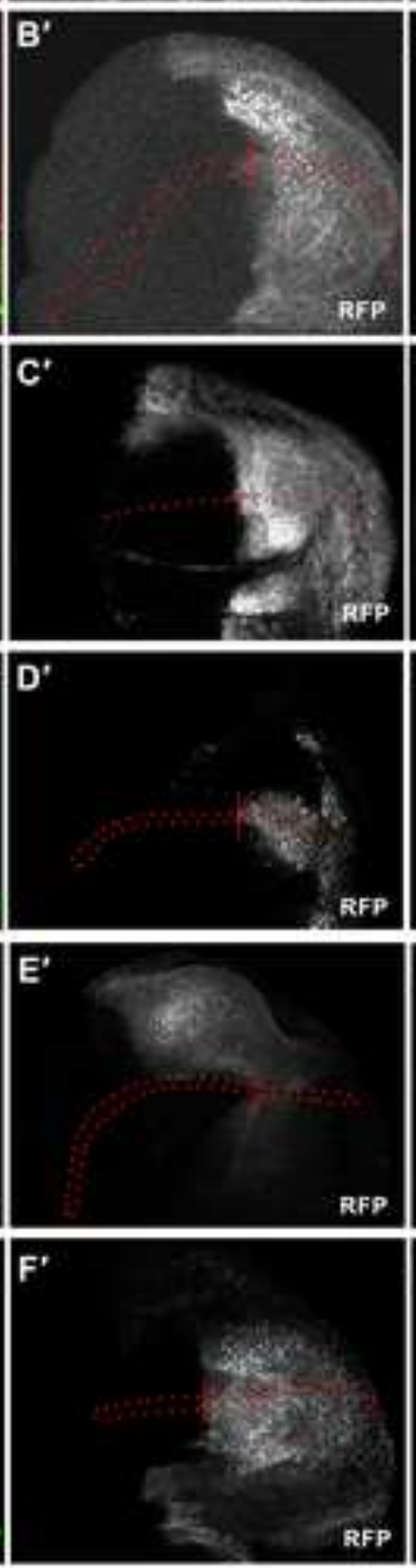

Ecdysone

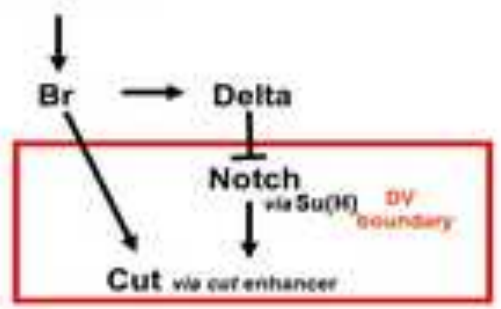

we car entianer

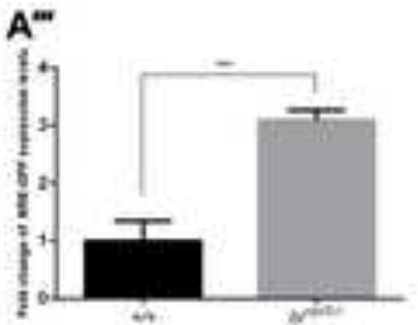

NRE-GFP
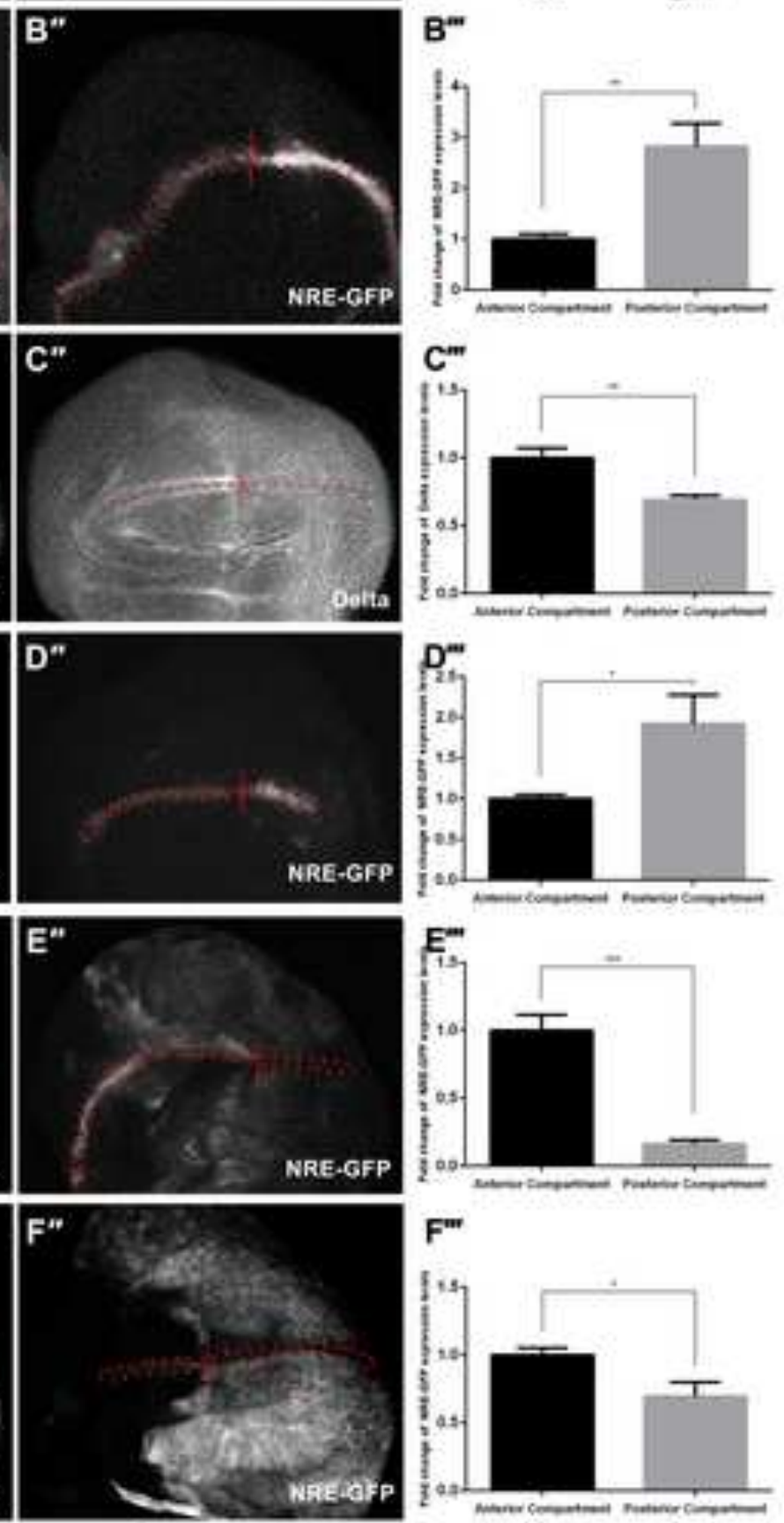